\title{
Heating of beam ions by ion acoustic waves
}

\author{
A. Vaivads, K. Rönnmark, T. Oscarsson, and M. André \\ Swedish Institute of Space Physics, University of Umeå, S-901 87 UMEÅ, Sweden
}

Received: 30 April 1997 / Revised: 25 August 1997 / Accepted: 2 September 1997

\begin{abstract}
Satellite measurements show that ion beams above the auroral acceleration region are heated to hundreds of $\mathrm{eV}$ in a direction perpendicular to the magnetic field. We show that ion acoustic waves may be responsible for much of this heating. Even in the absence of a positive slope in the velocity distribution of the beam ions, ion acoustic waves can be generated by a fan instability. We present analytical estimates of the wave growth rate and ion beam heating rate. These estimates, which are confirmed by particle simulations, indicate that the perpendicular temperature of the beam ions will increase by $30 \mathrm{eV} / \mathrm{s}$, or by $1 \mathrm{eV}$ in $20-25 \mathrm{~km}$. From the simulations we also conclude that the heating saturates at a perpendicular temperature around $200 \mathrm{eV}$, which is consistent with observations.
\end{abstract}

Key words. Ionosphere (wave-particle interactions) · Magnetospheric Physics (plasma waves and instabilities) - Space plasma physics (wave-particle interactions).

\section{Introduction}

Energetic upflowing field-aligned ion beams with $\mathrm{keV}$ energies are a frequently occurring phenomenon in the auroral zone. There are many statistical studies of the ion beam drift energies, composition and occurrence frequencies depending on factors such as solar activity, altitude, magnetic local time and invariant latitude (Kondo et al., 1990; Collin et al., 1987; Yau et al., 1984; Gorney et al., 1981). The ion beams are probably

Correspondence to: A. Vaivads accelerated by a quasi-static electric field parallel to the ambient magnetic field (Reiff et al., 1988). Nevertheless, the shape of the ion beam distributions cannot be explained by a parallel quasi-static electric field alone.

Energetic ion beams created only by acceleration of a background (ionospheric) ion component in a parallel electric field would show no change in the perpendicular temperature of the beams, and a decrease in the parallel temperature (the temperature defined from the average energy of beam ions in the beam reference system). However, satellite measurements indicate that ion beams are heated parallel to the magnetic field (Reiff et al., 1988), and also perpendicularly to the magnetic field (Kaufmann et al., 1986). The commonly accepted view is that ion beams are heated as a result of waveparticle interactions. However, there are different explanations concerning which waves are responsible for the wave-ion interactions and which are the sources of the waves.

Different waves have been observed in the ion beam regions. Electrostatic hydrogen cyclotron (EHC) waves (waves with frequencies above the hydrogen gyrofrequency) (Cattell et al., 1991) are found to be common. These waves, depending on their wave vectors, can cause both perpendicular and parallel heating of the beam. In addition, observations of electric wave fields at lower frequencies are also common. These emissions, sometimes referred to as low frequency turbulence, have maximum power spectral density at frequencies much below the ion gyrofrequency. The wave power decreases with increasing frequency but may still be significant above the proton gyrofrequency. The properties of these low frequency waves are not well known. The emissions may at least partly be due to Alfvén waves, possibly causing perpendicular ion energization. There may also be a significant component of electrostatic cyclotron waves related to heavier ions (oxygen and helium) (Cattell et al., 1991). The low frequency waves can also be ion acoustic waves (Wahlund et al., 1994). In this case, waves with a nearly parallel wave vector can cause ion beam heating parallel to the magnetic field. In 
addition, as we will show in this article, ion acoustic waves with a nearly perpendicular wave vector can cause perpendicular heating of the beam ions.

The source of the EHC and ion acoustic waves discussed is usually assumed to be some local instability. There are several types of instabilities that can develop. The source of EHC waves can be a drift of low energy electrons (often detected as a current) (Cattell et al., 1991) or an ion beam drift with respect to some background ions (André et al., 1987). Similarly, an electron drift or an ion beam drift can be the source of ion acoustic waves (André et al., 1987). In cases of both hydrogen and oxygen beams, the relative drift between different ion species can destabilize waves with frequencies above and below the proton gyrofrequency (Bergmann et al., 1988). During a particular event, the observed waves may be generated by a combination of instabilities.

Some instabilities are strongly dependent on the presence of a cool (eV) ion background component. It has been shown that at least in some ion beam regions such a cool background component can be present (Koskinen et al., 1990). However, due to observational difficulties, the amount of cool ion data obtained directly with particle instruments within ion beam regions is limited. As an alternative, sometimes indirect measurements can be used to determine whether cool ions are present in a plasma. For example, the difference in densities between electrons and ions detected by particle instruments can indicate the presence of a cool background plasma (André et al., 1987). While there is no statistical study of cool ions in regions of ion beams, a cool ion background may well be a common feature.

One way to check the consistency of a model explaining both wave generation and ion beam heating is to use numerical simulations. There have been several such studies (Gray et al., 1990; Schriver et al., 1990; Winglee et al., 1989). Most of these studies are concerned with instabilities generated by the relative drift between oxygen and hydrogen beams. It is found that when ion beams are subsonic the ions are heated parallel to the magnetic field, but when the ion beams are supersonic the heating is mostly perpendicular to the magnetic field. Some simulations have been performed to analyze systems consisting of background ions and an ion beam. One simulation included EHC wave generation and subsequent ion beam diffusion in both the perpendicular and the parallel directions (Miura et al., 1983). We also investigate wave generation and ion beam diffusion, but in our simulation low frequency ion acoustic waves are caused by a fan instability generated by an ion distribution with a cool background and a suprathermal tail.

The fan instability is based on the principle that a particle beam, or just a suprathermal tail in the particle distribution, through Doppler resonance can generate waves with phase velocities much lower than the beam drift velocity. Note that this instability is different from many other wave generation mechanisms related to upflowing particles in that no positive slope in the ion velocity space distribution is needed. The generated emissions can be electrostatic waves with a wave vector at large angles with respect to the ambient magnetic field. The fan instability is well known from studies of waves in fusion plasma devices, where a suprathermal tail in the electron distribution is created by current drive (Fuchs et al., 1988). It has been shown that auroral electron beams in a similar way can support fan instabilities generating lower hybrid waves (Omelchenko et al., 1994). It has also been shown that ion beams accelerated in the auroral acceleration region can similarly be unstable to the fan instability and generate ion acoustic waves (Vaivads et al., 1995). However, the nonlinear evolution of this instability has not been analyzed.

We also show that the quasilinear evolution of the fan instability is consistent with satellite observations. As an example of observations, we present a typical ion beam event detected by the Viking satellite. We show that the growth rate of the fan instability is large enough for the ion acoustic waves to develop high amplitudes. In addition, we show that these waves can efficiently heat ion beams perpendicularly to the magnetic field. The ion beam heating rate estimated from quasi linear theory is compared with simulations. We show that when waves observed in ion beam regions are ion acoustic waves, the wave amplitudes are high enough to explain the observed perpendicular heating of the ion beam. In addition, the results from our simulations show that the saturation of the ion beam perpendicular heating agrees well with satellite observations.

\section{Observations}

On May 21, 1986, the polar orbiting Viking satellite (Hultqvist, 1990) spent $6 \mathrm{~min}$ in a region of upgoing ion beams at an altitude of about $11000 \mathrm{~km}$ in the auroral zone. The ion beam region was found at local times between 14:00 and 15:00 MLT, and at invariant magnetic latitudes between $78^{\circ}$ and $80^{\circ}$. Figure 1 shows spectrograms of the waves and particles observed in and near the beam region. The ion spectrogram was obtained from measurements by two electrostatic analyzers mounted at $90^{\circ}$ with respect to the spacecraft spin axis, covering the energy ranges 0.04-1.2 keV and 1.2-40 keV, respectively (Sandahl et al., 1985). The ion beams are seen as vertical stripes near $180^{\circ}$ pitch angle (upgoing direction) in Fig. 1. The electron spectrometer is also mounted at $90^{\circ}$ relative to the spin axis and covers the energy range $30 \mathrm{eV}$ to $40 \mathrm{keV}$. The measuring cycle time for both electrons and positive ions is $0.30 \mathrm{~s}$. The electric field measurements are obtained from a pair of probes on the $40 \mathrm{~m}(80 \mathrm{~m}$ tip-to-tip) radial wire booms (Gustafsson et al., 1990). A 10 s modulation in the spectrogram is induced by the satellite spin. In the following we investigate the particle and wave observations, and how they are correlated.

The ion beam characteristics are typical for auroral regions. The beam drift energies are in the interval between about $200 \mathrm{eV}$ and $1 \mathrm{keV}$. The beams are aligned with the ambient magnetic field, having an angular half 
width of the order of 20 degrees. A closer investigation shows that the ion beams with lower drift velocity have a smaller angular width. We can estimate the ion beam perpendicular energy to be between a few tens of $\mathrm{eV}$, for ion beams with a drift energy of order $200 \mathrm{eV}$, and up to $200 \mathrm{eV}$ for ion beams with a drift energy of nearly $1 \mathrm{keV}$. The electron spectrogram shows the presence of electrons with energies of about $200 \mathrm{eV}$. From the absence of downward accelerated electrons we conclude that the satellite is located above the auroral acceleration region. This is also consistent with the widened electron loss cone that can be observed during several satellite spins.

The density in the ion beam region can be estimated in different ways. The most reliable method available may be to use relaxation sounder data (Bahnsen et al., 1988) to obtain the plasma frequency. This gives densities of $0.1-0.5 \mathrm{~cm}^{-3}$. A first estimate using Langmuir probe measurements gives densities of $2-4 \mathrm{~cm}^{-3}$ (A. Eriksson, private communication), and from the electron data we determine the density to be of the order of $1 \mathrm{~cm}^{-3}$ or less. These latter estimates are still consistent with a total density of $0.1-0.5 \mathrm{~cm}^{-3}$, especially since Langmuir probe measurements may be affected by photoelectron contamination. The density of the ion beam estimated from particle data is to be about $0.03 \mathrm{~cm}^{-3}$, or maybe somewhat lower. This means that the ratio between the beam and total densities is of the order of 0.1 , which may indicate the presence of cool ions.

The electric field spectrogram in the top panel of Fig. 1 shows that there is a general increase in electric field spectral density in the ion beam region. In addition, it is possible to distinguish between two regions of wave activity: emissions with a peak at frequencies between 40 to $60 \mathrm{~Hz}$ (high frequency, including the proton gyrofrequency at $45 \mathrm{~Hz}$ ), and waves with frequencies below about $10 \mathrm{~Hz}$ (low frequency). The wave amplitude in the beam region is of the order $10 \mathrm{mV} / \mathrm{m}$ for the high frequency waves. For the low frequency waves the amplitude can reach $100 \mathrm{mV} / \mathrm{m}$. From a more detailed investigation (not shown here) it is clear that when the electric field spectrogram shows spin modulations, the highest wave amplitudes occur when the booms are nearly perpendicular to the ambient magnetic field. The fact that the electric field is essentially perpendicular inside the ion beam region is of interest when comparing with the simulations presented in the following.

Our simulations are based on a plasma model consistent with the event described already. Such events are common, and our model is also consistent with other Viking ion beam events (André et al., 1987; Vaivads et al., 1995). Hence, the plasma model used in our analysis and simulations describes a typical ion beam region above the auroral acceleration region.

VIKING, Orbit 485, date: 860521

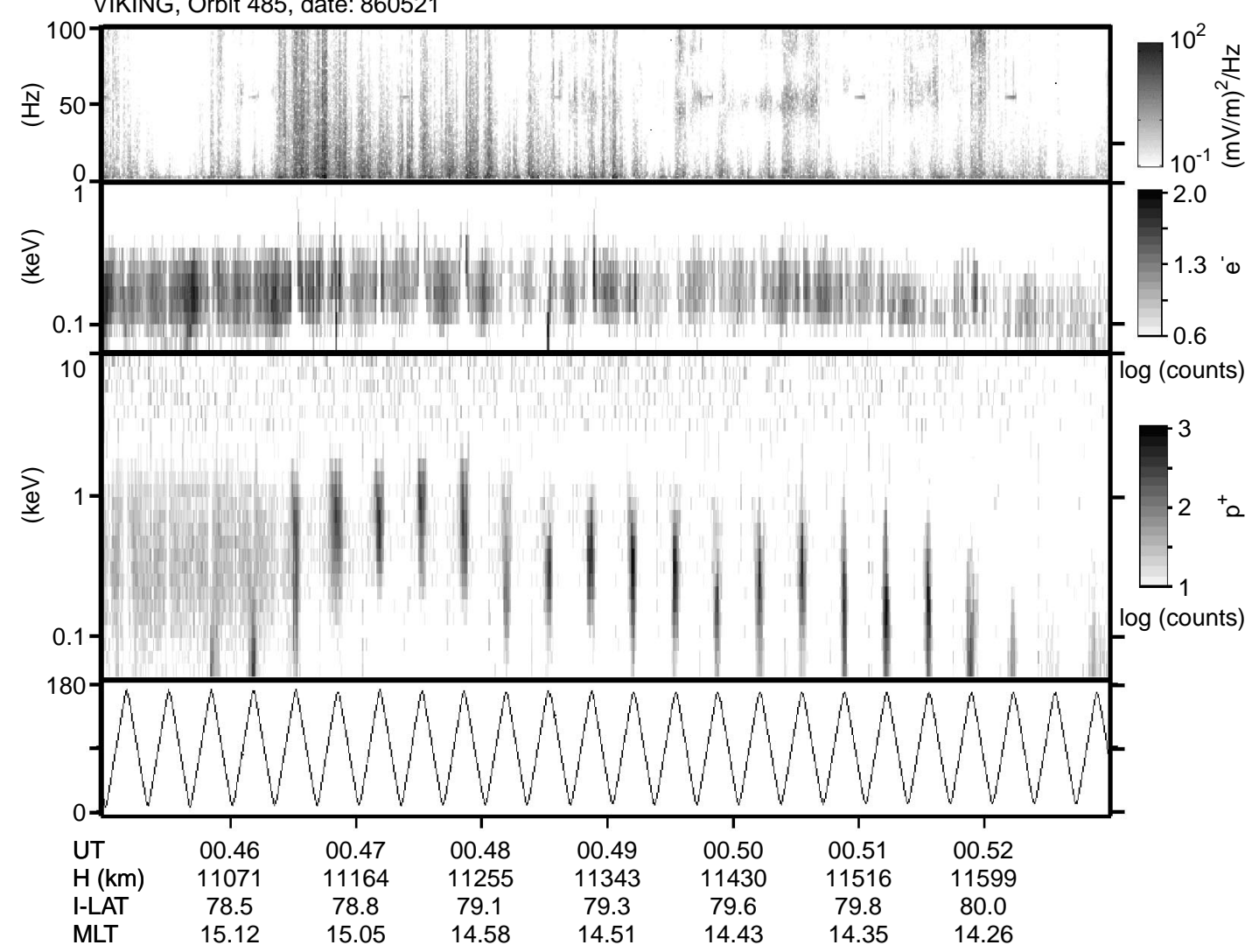

Fig. 1. The four panels show (from above): 1, electric field spectrogram, satellite spin modulation is clearly seen; 2, electron spectrogram; 3, proton spectrogram; 4, pitch angle of the particle detectors with respect to the magnetic field 


\section{Theory}

The fan instability is driven by a particle beam along the magnetic field, but in contrast to the usual beam-plasma instabilities it does not require a positive slope in the particle distribution function. The theory of the fan instability is best illustrated in the electrostatic approximation. We start from the dielectric function $\varepsilon(\omega, \mathbf{k})$ of a plasma consisting of several particle species $\sigma$ with distribution functions $f \sigma\left(v_{\|}, v_{\perp}\right)$

$$
\begin{aligned}
\varepsilon= & 1-\sum_{\sigma} \frac{\omega_{\sigma}^{2}}{k^{2}} \sum_{n=-\infty}^{\infty} \int \mathrm{d} \mathbf{v} \frac{J_{n}^{2}\left(\frac{k_{\perp} v_{\perp}}{\Omega_{\sigma}}\right)}{n \Omega_{\sigma}+k_{\|} v_{\|}-\omega} \\
& \times\left(\frac{n \Omega_{\sigma}}{v_{\perp}} \partial v_{\perp} f \sigma+k_{\|} \partial v_{\|} f \sigma\right),
\end{aligned}
$$

where $\omega_{\sigma}$ and $\Omega_{\sigma}$ are the plasma frequency and gyro frequency of plasma component $\sigma$, and $J_{n}$ is an ordinary Bessel function. When calculating the real part $\varepsilon_{R e}$ of the plasma that consists of hot electrons $(\sigma=e)$ with thermal velocity $V_{e} \gg \omega / k$ and protons $(i)$ that here can be treated as cold. This leads to

$\varepsilon_{R e}(\omega, \mathbf{k})=1+2 \frac{\omega_{e}^{2}}{k^{2} V_{e}^{2}}-\frac{\omega_{i}^{2}}{k^{2}}\left(\frac{k_{\|}^{2}}{\omega^{2}}+\frac{k_{\perp}^{2}}{\omega^{2}-\Omega_{i}^{2}}\right)$

The growth rate $\gamma$ of the waves is found from the imaginary part $\varepsilon_{I m}$ of the dielectric function as

$\gamma=-\frac{\omega \varepsilon_{I m}}{\omega \partial_{\omega} \varepsilon_{R e}}$.

The condition for instability $(\gamma>0)$ of positive energy waves $\left(\omega \partial_{\omega} \varepsilon_{R e}>0\right)$ is thus that $\omega \varepsilon_{I m}(\omega, \mathbf{k})<0$ for a frequency that satisfies the dispersion relation $\varepsilon_{R e}(\omega, \mathbf{k})=0$. When calculating $\varepsilon_{I m}$ we must include the contribution from the resonant particles. Assuming the proton distribution to have the form

$f_{i}\left(v_{\|}, v_{\perp}\right)=F_{i}\left(v_{\|}\right) \pi^{-1} V_{i \perp}^{-2} \exp \left[-\frac{v_{\perp}^{2}}{V_{i \perp}^{2}}\right]$,

and considering wavelengths larger than the ion gyro radius $\left(k_{\perp} V_{i \perp} / \Omega_{i}<1\right)$, we can integrate over $v_{\perp}$ and use the small argument expansion for the Bessel functions. keeping only the resonant contributions from the poles in the integration over $v_{\|}$and the $n=0, \pm 1$ terms in the sum over harmonic numbers we find dielectric function, we consider low frequency waves in a

$$
\begin{aligned}
\gamma= & \frac{\pi}{2} \frac{k_{\|}}{\left|k_{\|}\right|} \omega\left(\frac{k_{\|}^{2}}{\omega^{2}}+\frac{k_{\perp}^{2} \omega^{2}}{\left(\omega^{2}-\Omega_{i}^{2}\right)^{2}}\right)^{-1} \\
& \times\left\{\frac{m_{i}}{m_{e}} F_{e}^{\prime}\left(\frac{\omega}{k_{\|}}\right)+F_{i}^{\prime}\left(\frac{\omega}{k_{\|}}\right)\right. \\
& +\frac{k_{\perp}^{2}}{2 \Omega_{i}} \frac{V_{i \perp}^{2}}{2 \Omega_{i}} F_{i}^{\prime}\left(\frac{\omega+\Omega_{i}}{k_{\|}}\right) \\
& +\frac{k_{\perp}^{2}}{2 \Omega_{i}} \frac{V_{i \perp}^{2}}{2 \Omega_{i}} F_{i}^{\prime}\left(\frac{\omega-\Omega_{i}}{k_{\|}}\right) \\
& +\frac{k_{\perp}^{2}}{2 \Omega_{i}} \frac{1}{k_{\|}} F_{i}\left(\frac{\omega+\Omega_{i}}{k_{\|}}\right) \\
& \left.-\frac{k_{\perp}^{2}}{2 \Omega_{i}} \frac{1}{k_{\|}} F_{i}\left(\frac{\omega-\Omega_{i}}{k_{\|}}\right)\right\}
\end{aligned}
$$

When the parallel distributions $F_{\sigma}$ have a single maximum at $v_{\|}=0$ and $\omega>\Omega_{i}$, the first four terms, containing the derivative $F_{\sigma}^{\prime}=\partial_{v \|} F_{\sigma}$, will all be negative and contribute to damping. The last term will obviously always be negative, and it will in this case dominate the only positive term Eq. (5d). Hence, we expect no instabilities at frequencies above the gyrofrequency. However, when the wave frequency is less than the gyrofrequency, the term (5c) will become positive, and if $F_{i}$ is sufficiently asymmetric the term $(5 \mathrm{~d})$ can be much larger than Eq. (5e). Under these conditions we may have a fan instability.

A situation that is favourable for ion fan instability is shown in Fig. 2. The ion distribution function sketched in this figure has a single maximum at $v_{\|}=0$, but it is very asymmetric with a long tail in the direction of positive $v_{\|}$. The relevant resonant velocities are indicated for the case $\omega<\Omega$. At the velocity $v_{L}=\omega / k_{\|}$corresponding to Landau resonance the distribution is almost flat, and the ion Landau damping is thus negligible. The contributions from the resonance at $v_{-}=\left(\omega-\Omega_{i}\right) / k_{\|}$ will also be small, since both $F_{i}\left(v_{-}\right)$and $F_{i}^{\prime}\left(v_{-}\right)$are small. If the tail extends out to the resonance at $v_{+}=\left(\omega+\Omega_{i}\right) / k_{\|}$so that $F_{i}\left(v_{+}\right)$is reasonably large, the contribution from the term (5d) may dominate and cause an ion fan instability.

In the presence of electrostatic waves with a spatially homogeneous k-spectrum $|\mathbf{E}(\mathbf{k})|^{2}$, the quasi-linear diffusion of resonant particles will make the particle distribution evolve as (e.g. Shapiro and Shevchenko, 1988)

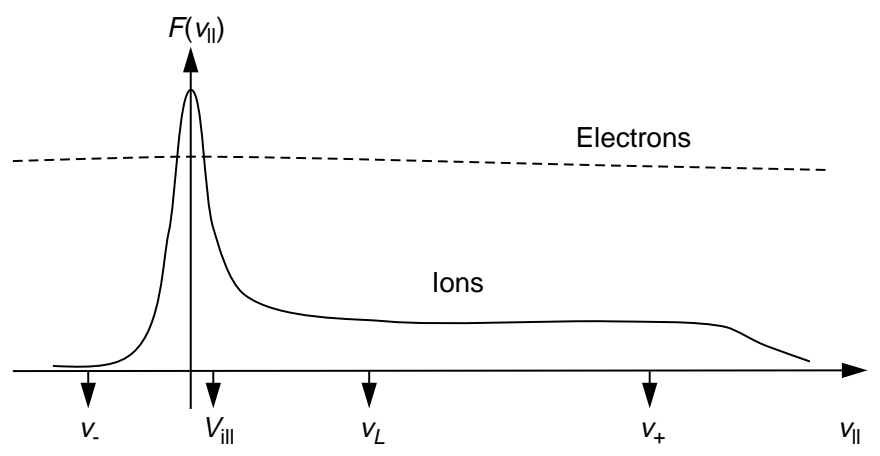

Fig. 2. Schematic picture of the ion distribution and the location of resonant velocities, $v_{L}=\omega / k_{\|}, v_{+}=\left(\omega+\Omega_{i}\right) / k_{\|}, v_{-}=\left(\omega-\Omega_{i}\right) / k_{\|}$. The thermal velocity of cold ions is $V_{i \|}$ 


$$
\begin{aligned}
\partial_{t} f_{0}(\mathbf{v})= & \frac{e^{2}}{8 \pi^{2} m^{2}} \int \mathrm{d} \mathbf{k} \sum_{n=-\infty}^{\infty}\left(\frac{n \Omega_{i}}{v_{\perp}} \partial_{v \perp}+k_{\|} \partial_{v \|}\right) \\
& \times k^{-2}|\mathbf{E}(\mathbf{k})|^{2} J_{n}^{2}\left(\frac{k_{\perp} v_{\perp}}{\Omega_{i}}\right) \delta\left(\omega(\mathbf{k})-n \Omega_{i}-k_{\|} v_{\|}\right) \\
& \times\left(\frac{n \Omega_{i}}{v_{\perp}} \partial v_{\perp}+k_{\|} \partial_{v \|}\right) f_{0} .
\end{aligned}
$$

Since we intend to compare with one-dimensional simulations, we assume the spectrum to have the form

$$
|\mathbf{E}(\mathbf{k})|^{2}=\left|\mathbf{E}\left(k_{x}\right)\right|^{2} \delta\left(k_{y}\right) \delta\left(k_{z}\right) \text {. }
$$

The Cartesian coordinate system is here chosen so that the ambient magnetic field is in the $x-z$ plane at an angle $\theta$ from the $z$-axis. With this model, we can evaluate the integral over $\mathbf{k}$-space to obtain

$$
\begin{aligned}
\partial_{t} f_{0}(\mathbf{v})= & \frac{e^{2}}{8 \pi^{2} m^{2}} \sum_{n=-\infty}^{\infty}\left(\frac{n \Omega_{i}}{v_{\perp}} \partial_{v \perp}+\partial_{v \|} k_{\|}\right) \\
& \times \frac{|\mathbf{E}(k)|^{2}}{\left|v_{\|} \cos \theta-\partial_{k} \omega\right|} \frac{J_{n}^{2}\left(\frac{k_{\perp} v_{\perp}}{\Omega_{i}}\right)}{k^{2}} \\
& \times\left(\frac{n \Omega_{i}}{v_{\perp}} \partial_{v_{\perp}}+k_{\|} \partial_{v_{\|}}\right) f_{0} .
\end{aligned}
$$

where $k=\left(\omega-n \Omega_{i}\right) /\left(v_{\|} \cos \theta\right), k_{\|}=k \cos \theta$, and $k_{\perp}=$ $k \sin \theta$. Concentrating on the beam ions that satisfy the resonance condition $v_{\|} \sim\left(\omega+\Omega_{i}\right) / k_{\|}$, we keep only the term with $n=-1$. The diffusion will be mainly in the perpendicular direction, where the gradients in velocity space are strong. The equation for perpendicular velocity diffusion is

$\partial_{t} f_{0}(\mathbf{v})=\frac{1}{v_{\perp}} \partial_{v \perp} D_{v \perp} \partial_{v \perp} f_{0}$,

where the diffusion coefficient is given by

$D=\frac{e^{2}}{8 \pi^{2} m^{2}} \frac{|\mathbf{E}(k)|^{2} \Omega_{i}^{2}}{\left|v_{\|} \cos \theta-\partial_{k} \omega\right|} \frac{J_{1}^{2}\left(\frac{k_{\perp} v_{\perp}}{\Omega_{i}}\right)}{k^{2} v_{\perp}^{2}}$.

Since we will consider waves with $k_{\perp} V_{i \perp} / \Omega_{i}<1$, we can use the small argument limit of the Bessel function $J_{1}\left(k_{\perp} v_{\perp} / \Omega_{i}\right) \approx \frac{1}{2} k_{\perp} v_{\perp} / \Omega_{i}$, which simplifies the diffusion coefficient to

$D=\frac{e^{2}}{32 \pi^{2} m^{2}} \frac{|\mathbf{E}(k)|^{2} \sin ^{2} \theta}{\left|v_{\|} \cos \theta-\partial_{k} \omega\right|}$.

Within this approximation, the diffusion coefficient is independent of $v_{\perp}$, and we find that a distribution function of the form

$f_{i}\left(v_{\|}, v_{\perp}, t\right)=\frac{m F_{i}\left(v_{\|}\right)}{\pi 2 T_{\perp}} \exp \left[-\frac{m v_{\perp}^{2}}{2 T_{\perp}}\right]$

satisfies the diffusion Eq. (9), provided that the perpendicular temperature $T_{\perp}(t)$ satisfies

$\partial_{t} T_{\perp}=2 m D$.

\section{Method}

The code used in our simulations is adapted from the code es1 (Birdsall and Langdon, 1991), which is an electrostatic particle simulation code with one space and two velocity dimensions. In our version of the code, the ions are treated as particles with three velocity components, while electrons contribute only through the Debye screening (see also Okuda et al., 1978). The electric field that interacts with the ions is found by solving Poisson's equation,

$\left(k^{2}+k_{e}^{2}\right) \phi=e\left(n_{i}-n_{0}\right) / \varepsilon_{0}$,

where $k_{e}^{2}=n_{0} e^{2} / \varepsilon_{0} T_{e}$ is the electron Debye wave vector, $n_{i}$ is the ion density, and $n_{0}$ is the average electron density, and $T_{e}$ is the electron temperature. As a result, we can investigate electrostatic ion waves, including ion acoustic waves, propagating in a specified direction with respect to the background magnetic field without resolving the faster electron time scale.

The ion distribution consists of a background Maxwellian distribution with density $n_{i M}=0.45 \mathrm{~cm}^{-3}$ and temperature $1 \mathrm{eV}$, and an ion beam with density $n_{i b}=0.05 \mathrm{~cm}^{-3}$. Our code allows us to specify the initial ion beam distribution as a plateau with Maxwellian tails,

$$
\begin{aligned}
F_{i b}\left(v_{\|}\right)= & \frac{n_{i b} / n_{0}}{\left(V_{i l}+V_{i h}\right) \sqrt{\pi} / 2+\vartheta_{h}-\vartheta_{l}} \\
& \times \begin{cases}\exp -\frac{\left(v_{\|}-\vartheta_{l}\right)^{2}}{V_{i l}^{2}}, & v_{\|}<\vartheta_{l} ; \\
1, & \vartheta_{l}<v_{\|}<\vartheta_{h} ; \\
\exp -\frac{\left(v_{\|}-\vartheta_{h}\right)^{2}}{V_{i h}^{2}}, & \vartheta_{h}<v_{\|} ;\end{cases}
\end{aligned}
$$

where $\vartheta_{l}=0, m V_{i l}^{2} / 2=1 \mathrm{eV}, m \vartheta_{h}^{2} / 2=2500 \mathrm{eV}$, and $m V_{i h}^{2} / 2=400 \mathrm{eV}$. Initially, all the ions have a perpendicular temperature of $1 \mathrm{eV}$, and hence $V_{i \perp}=$ $V_{i l}=13841 \mathrm{~m} / \mathrm{s}$.

In the simulations, the Maxwellian background is represented by 131072 particles, and the beam by 524288 particles. The angle between the simulation system and the magnetic field in chosen to $80^{\circ}$. This is approximately the angle of maximum growth rate of the fan instability (Vaivads et al., 1995). The system length is $235 \mathrm{~km}$, which means that the length of a field line going through the simulation system at an angle of $80^{\circ}$ is about $1350 \mathrm{~km}$. The number of grid points is 4096, and the cell size is $57 \mathrm{~m}$. Electron distributions above the auroral acceleration region have typical temperatures of a few hundred $\mathrm{eV}$. In our simulations we have used $\lambda_{e}=k_{e}^{-1}=123 \mathrm{~m}$, which corresponds to an electron temperature of $136 \mathrm{eV}$, or as in the study by André et al. (1987), to a mixture of $94 \% 700 \mathrm{eV}$ and $6 \% 10 \mathrm{eV}$ electrons. The time step we use is $0.1 / \Omega_{i}$, where the ion gyrofrequency $\Omega_{i}=2 \pi \cdot 45 \mathrm{~Hz}$.

\section{Results}

When we start the simulation with the ion distribution as described, the energy in the electric field fluctuations 


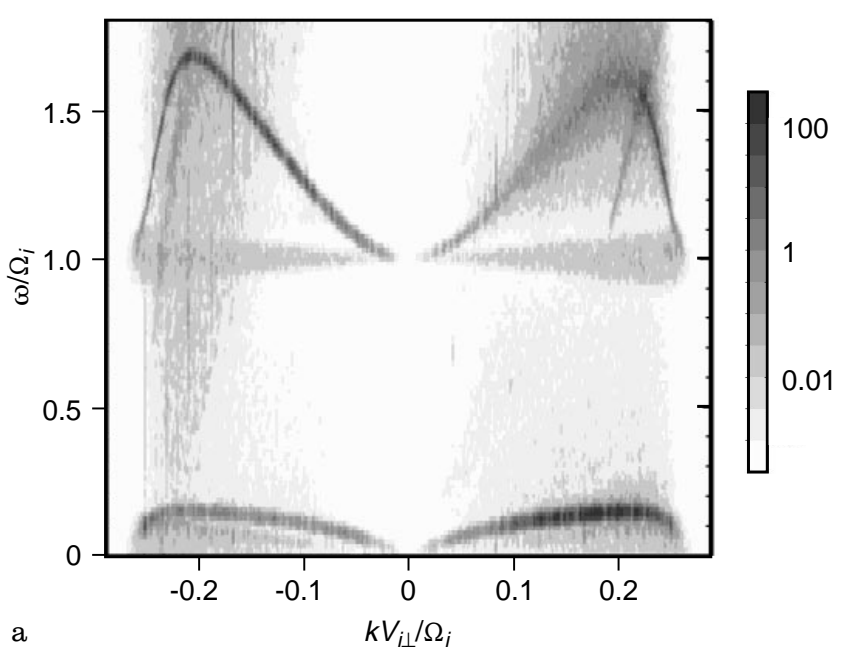

Fig. 3. a The distribution of wave-power in the frequency-wave vector plane in arbitrary units. Most of the wave-power is located where the plasma dispersion relation is satisfied. Ion acoustic waves can be seen

is about $10^{-5}$ times the total energy in the plasma. There is no prescribed perturbation, and the plasma waves grow from this noise level. The distribution of wave energy in the $\omega$-k plane obtained from the first $2.9 \mathrm{~s}$ of simulation is shown in Fig. 3a. For comparison we show in Fig. $3 b$ the dispersion surfaces, of the relevant modes, obtained with the WHAMP code (Rönnmark, 1983). A heavy line indicates a propagation angle of $80^{\circ}$. As expected, most of the wave energy is found close to the dispersion surfaces, where the fluctuations are weakly damped. At large $k$, i.e., $k V_{i \perp} / \Omega_{i}>=0.25$, we have artificially introduced a filter in the simulations that removes short wavelength fluctuations. To some extent, this filtering mimics the effects of electron Landau damping. We do not expect the resulting modification of the dispersion relation at large $k$ to influence the results presented below.

The wave energy concentration at frequencies just above $\sim 0.1 \Omega_{H+}$ in the ion acoustic mode is caused by the ion fan instability. The growth rate expected from the fan instability can be calculated from Eq. (5). There is no electron Landau damping in the simulations, since the electrons are represented as a fluid. To allow comparison with the simulations, the electron Landau damping term (5a) is neglected in the analytical calculation. With the ion distribution function used in the simulations only the term (5d) will contribute to the growth rate, and we find $\gamma=2.77 \cdot 10^{-3} \Omega_{i}$ at $k V_{i \perp} / \Omega_{i}=0.13$. To calculate the wave amplitudes from the growth rate, we notice that initially the signal is dominated by noise. Assuming that half of the noise consists of waves that are not growing because they propagate in the direction opposite to the beam ions, we expect the amplitudes to evolve as

$\left|A_{k}(t)\right|^{2}=\left|A_{k 0}\right|^{2}\left(1+e^{2 \gamma t}\right)$.

In Fig. 4, this function is shown together with the wave energy in the corresponding mode from the simulation. The growth rate from the simulation agrees

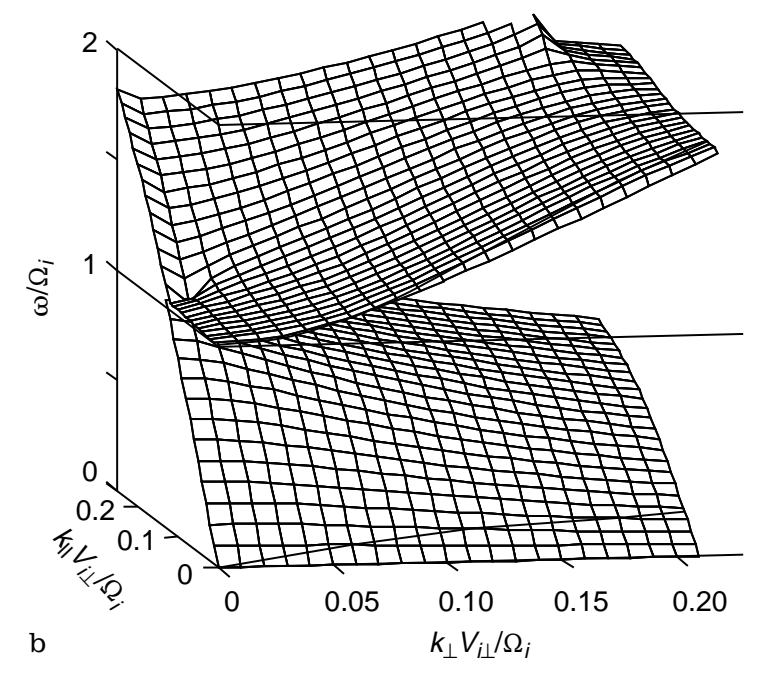

at frequencies below $0.2 \Omega_{i}$, and electrostatic ion cyclotron waves can be seen at frequencies above $\Omega_{i}$. b Numerical solution of the dispersion relations. The $80^{\circ}$ direction is marked with a solid line

well with the theoretical value over several seconds. In the simulation, the growth rate then decreases, mainly due to the increase in the perpendicular temperature of the beam, and the wave energy saturates almost four orders of magnitude above the noise level.

To facilitate the comparison of the diffusion rate in the simulation with the theoretical value, it is convenient to keep the wave amplitudes constant during the interaction with the beam ions. We have run a simulation without background ions and with prescribed wave fields that satisfy the ion-acoustic dispersion relation. Since the density in this run is very low, $n_{i}=5 \cdot 10^{-6} \mathrm{~cm}^{-3}$, the fields generated by the particles can be neglected. The imposed fields are chosen such that waves with mode numbers between 65 and 130 are excited with amplitudes $A_{k}^{2}=0.67(\mathrm{mV} / \mathrm{m})^{2}$ and random phases. The resulting electric field amplitude is about $6.6 \mathrm{mV} / \mathrm{m}$. In our one-dimensional system of

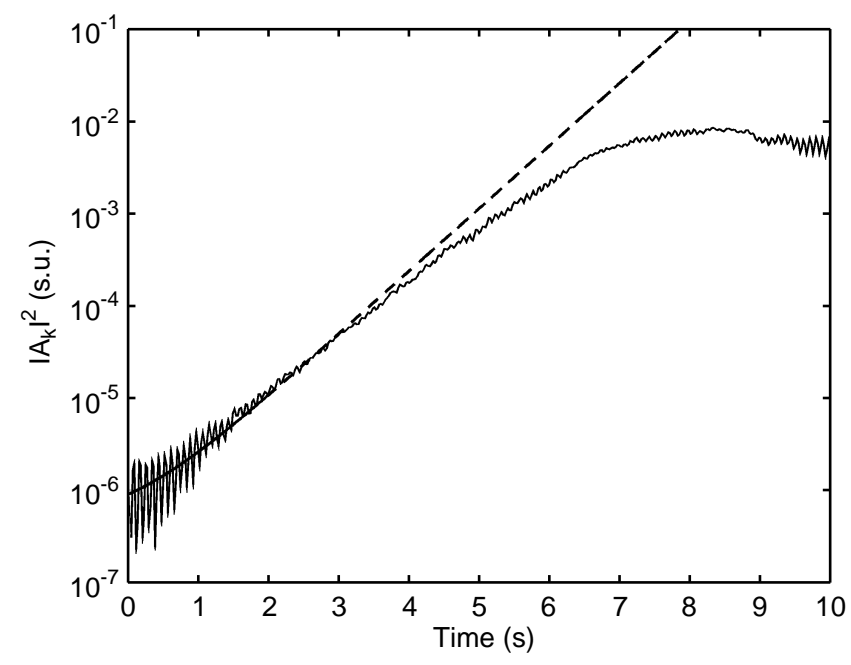

Fig. 4. The growth rate calculated form Eq. (5) (dashed), compared with the growth of the wave field in the simulation (solid line) 
length $L \approx 235 \mathrm{~km}$, these amplitudes are related to $|\mathbf{E}(k)|^{2}$ as

$|\mathbf{E}(k)|^{2}=4 \pi^{2} L\left|A_{k}\right|^{2}$

for $0.085<k V_{i} / \Omega_{i}<0.17$. Outside this range of wave numbers, the amplitudes are very low.

For ion acoustic waves on the resonance cone at $\theta \approx 80^{\circ}$, we find that the group velocity is small compared to $v_{\|} \cos \theta$. We can then, from Eq. (11), write the diffusion coefficient as

$D=\left(\frac{e}{m}\right)^{2} \frac{L\left|A_{k}\right|^{2} \sin ^{2} \theta}{8 v_{\|} \cos \theta}$.

Inserting the numbers $(e / m)^{2}=9.176 \cdot 10^{15}(\mathrm{As} / \mathrm{kg})^{2}$ and $\sin ^{2} 80^{\circ} /\left(8 \cos 80^{\circ}\right)=0.698$ we find $D=\left(V_{i \perp} / v_{\|}\right)$ $7.275 \cdot 10^{11} \mathrm{~m}^{2} \mathrm{~s}^{-3}$. This diffusion coefficient corresponds to the heating rate $\partial_{t} T_{\perp} \approx\left(V_{i \perp} / v_{\|}\right) 2.43 \cdot 10^{-16} \mathrm{~J} / \mathrm{s}$. At a parallel velocity $v_{\|}=\vartheta_{h}=50 V_{i \perp}$, corresponding to a beam ion energy of $2.5 \mathrm{keV}$, the heating rate is then

$\partial_{t} T_{\perp} \approx 30 \mathrm{eV} / \mathrm{s}$.

Alternatively, we can interpret this as a temperature increase with altitude at the rate $0.044 \mathrm{eV} / \mathrm{km}$, or $1 \mathrm{eV}$ in $23 \mathrm{~km}$.

A comparison between the theoretical heating rate given in Eq. (19) and the perpendicular temperature increase at $v_{\|}=\vartheta_{h}$ observed in the simulation is shown in Fig. 5. The theoretical estimate agrees very well with the simulation during the first five seconds. When the temperature is around $200 \mathrm{eV}$, the assumption $k_{\perp} v_{\perp} /$ $\Omega_{i}<1$ is violated for a large fraction of the particles, and the small argument expansion of the Bessel functions is no longer valid. We can then expect that our simplified theory will overestimate the heating rate. From Eq. (10) we expect the diffusion coefficient to go to zero as the perpendicular velocity increases so that $k_{\perp} v_{\perp} / \Omega_{i}$ approaches the first zero of $J_{1}$, which explains

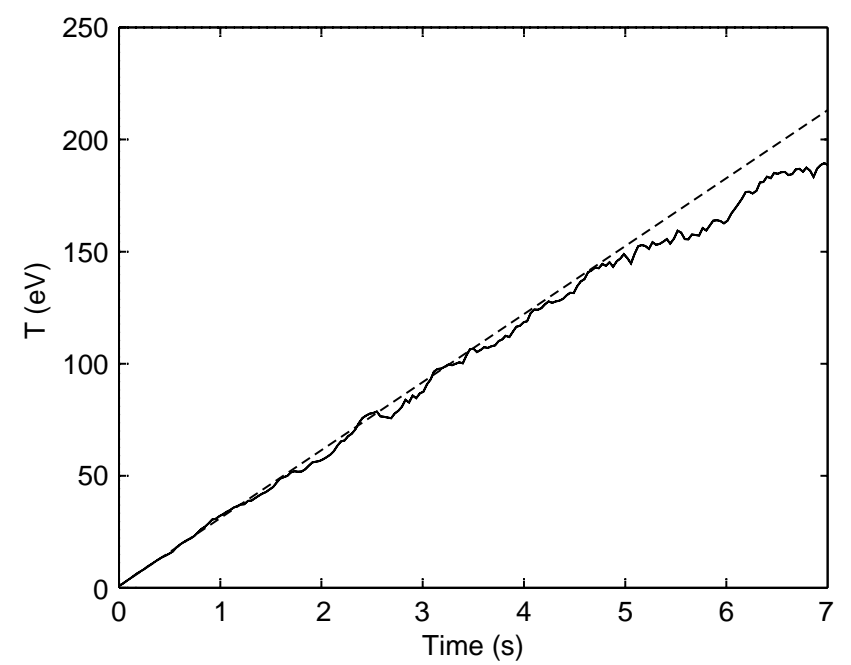

Fig. 5. The perpendicular temperature at $v_{\|}=\vartheta_{h}$ from the simulation with constant amplitudes (solid line), compared with the theoretical value from Eq. (19) (dashed)

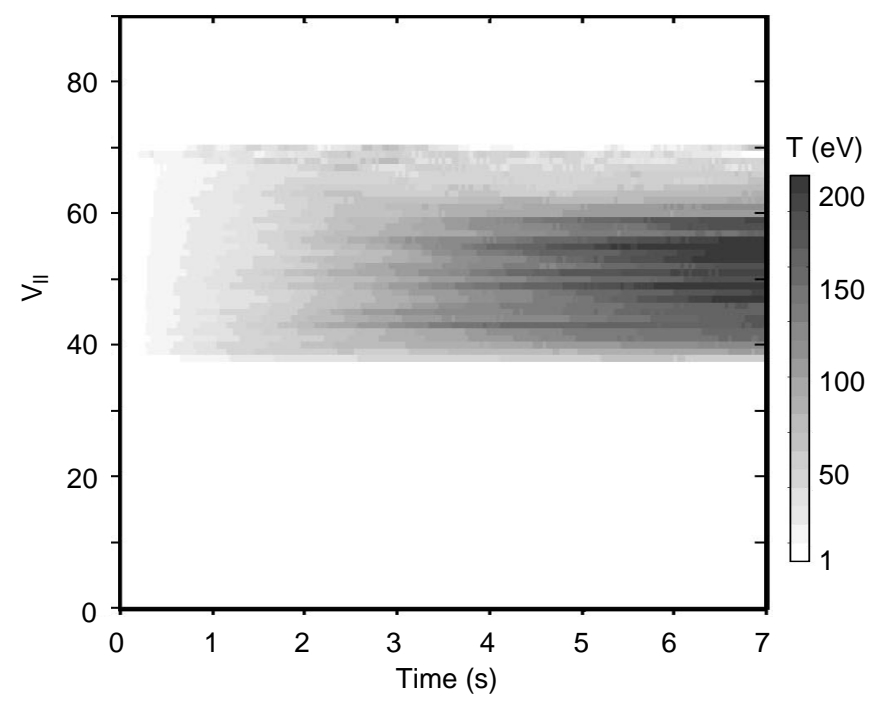

Fig. 6. Evolution of the perpendicular temperature at different parallel velocities

why the temperature in the simulation saturates at a few hundred $\mathrm{eV}$.

The evolution of the perpendicular temperature at different parallel velocities as a function of time is shown in Fig. 6. Since the excited spectrum covers parallel wave numbers satisfying approximately $0.015<k_{\|} V_{0 i} /$ $\Omega_{i}<0.03$, and the frequency $\omega \approx 0.125 \Omega_{i}$, we expect the resonance condition $v_{\|}=\left(\omega+\Omega_{i}\right) / k_{\|}$to be satisfied for parallel velocities in the range $37 V_{i \perp}<v_{\|}<75 V_{i \perp}$. Figure 6 confirms that the perpendicular temperature increases at these velocities. We also see that the heating rate decreases with increasing parallel velocity, as expected from Eq. (18).

We now return to the self-consistent simulations, with wave fields generated by the simulated particles. The wave amplitudes are then not constant but growing from noise. The evolution of the perpendicular temperature in this case is shown in Fig. 7. For comparison, we have also plotted the evolution of the perpendicular temperature predicted by integration of Eq. (13), using Eqs. (16) and (18),

$T(t)=T_{0}+2 m D_{0}\left(t+\frac{1}{2 \gamma}\left(e^{2 \gamma t}-1\right)\right)$.

where $D_{0}$ is determined from Eq. (18) with $\left|A_{k}\right|^{2}$ replaced by $\left|A_{k 0}\right|^{2}=4.5 \cdot 10^{-7}(\mathrm{mV} / \mathrm{m})^{2}$. The heating rate in the simulation is significantly lower than the theoretical prediction after about $4 \mathrm{~s}$, which is consistent with the decrease in the growth rate shown in Fig. 4.

As our final example we present in Fig. 8 a simulation run with a spatially inhomogeneous ion beam. The system length is in this case increased by a factor of four, so that $L=940 \mathrm{~km}$. Initially, the plateau distribution of ions is confined to the region $x<L / 2$. These ions later propagate to larger $x$, where a distribution with positive $\partial_{v \|} F_{i}$ is created. A phase-space plot of the distribution in the $x-v_{\|}$plane after $2 \mathrm{~s}$ is shown in Fig. 8a. The time series of the electric field, sampled at $x=3 L / 16$, 


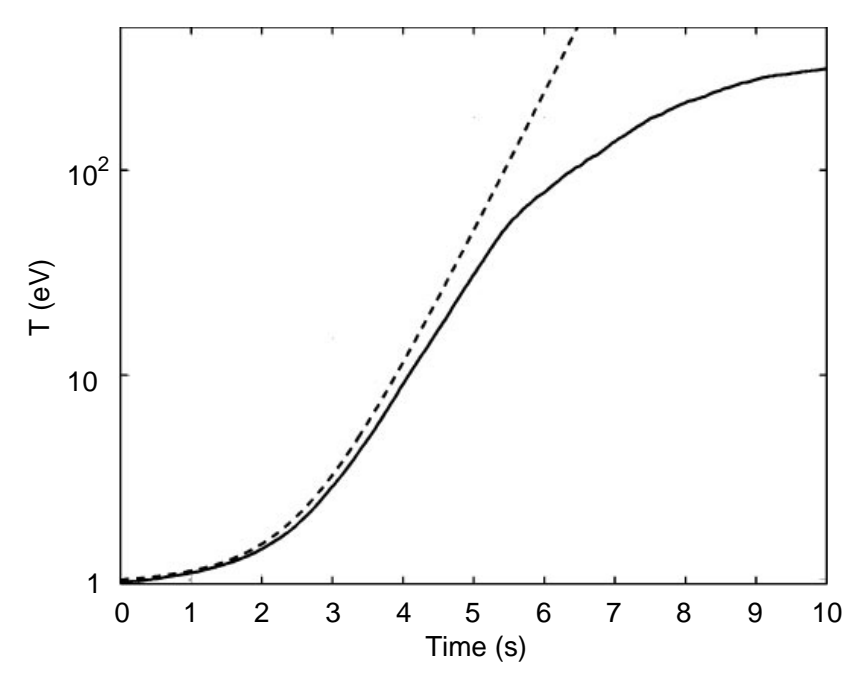

Fig. 7. The increase of the perpendicular temperature at $v_{\|}=\vartheta_{h}$ in the presence of a fan instability. The solid line shows the simulation result, and the dashed line the approximation in Eq. (20)

$7 L / 16,11 L / 16$, and $15 L / 16$ is shown in Fig. 8 b. At small $x$, the ion acoustic waves will initially grow due to the fan instability, but after about $2 \mathrm{~s}$ the high energy particles disappear and the growth stops. Near the middle of the system, at $x=7 L / 16$, where the energetic ions remain longer, the ion acoustic waves continue to grow until the end of the run. The stronger high frequency fluctuations at this point may be caused by ion cyclotron waves that propagate backwards from $x>L / 2$. When the fast ions reach $x=11 L / 16$, ion cyclotron waves with $\omega>\Omega_{i}$ start to grow rapidly. Although the amplitude modulation of these waves at first sight seems to be related to the ion acoustic waves, a closer inspection including points at slightly different $x$ values indicates that the amplitude fluctuations are random. Finally, at $x=15 L / 16$ near the end of the system, the ion cyclotron waves start to grow when the beam ions arrive after about $2.4 \mathrm{~s}$.

The perpendicular heating caused by these waves can be seen in Fig. 8c, which shows the ion density in the $x-v_{\perp}$ plane. The diffusion at $x<L / 2$ is caused by ion acoustic waves, generated by the fan instability. In our one-dimensional simulation, the higher frequency ion cyclotron waves also cause perpendicular diffusion, since they are forced to propagate almost perpendicular to the magnetic field. In three dimensions, nearly parallel propagating waves with $\omega>\Omega_{i}$ are expected to have the highest growth rates. These waves would then mainly cause parallel diffusion, and their main effect would be to flatten the positive slope in the parallel distribution to a plateau, rather than to cause perpendicular heating.

\section{Discussion}

Within the acceleration region, the parallel electric field will create an ion velocity distribution with a positive $v_{\|} \partial v_{\|} F_{i}\left(v_{\|}\right)$. This positive slope in the ion distribution will generate through Landau resonance, e.g. electrostatic hydrogen cyclotron (EHC) waves with frequencies around or above the ion gyrofrequency. These waves
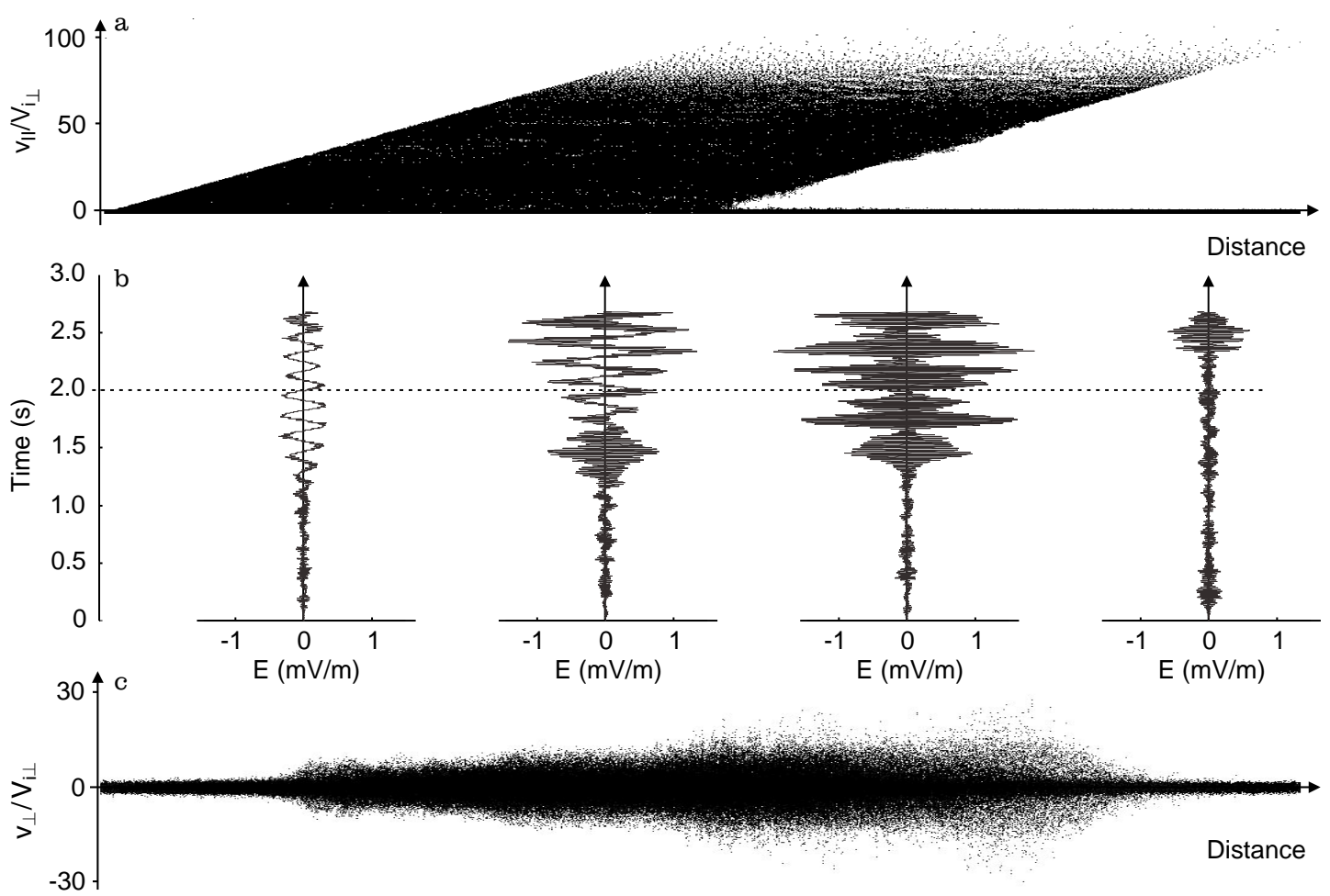

Fig. 8a-c. Inhomogenous beam simulation. a Shows simulation particles in $v_{\|}-x$ plane. b Shows the time evolution of the electric field at the marked positions. $\mathbf{c}$ Shows simulation particles in $v_{\perp}-x$ plane. a and $\mathbf{c}$ are snapshots after $2 \mathrm{~s}$ 
will cause parallel velocity diffusion that tends to remove the positive slope from the velocity distribution, but the slope will continuously be recreated by the electric field. Near the top of the acceleration region we can then expect the ion velocity distribution to have a long tail in the upward direction. When the positive slope on the tail is removed, this distribution is no longer unstable to EHC waves. However, oblique ion acoustic waves with frequencies below the ion gyrofrequency can still be generated by the fan instability.

To allow direct comparisons between quasi-linear theory and our one-dimensional simulations, we have for our analytical estimates assumed the waves to propagate strictly in one direction. Replacing Eq. (7) by a more realistic wave distribution over all azimuthal angles and some range of pitch angles $\theta$, we find that the only change in Eq. (8) is that the denominator $\left|v_{\|} \cos \theta-\partial_{k} \omega\right|$ is replaced by $\left|v_{\|} \cos \theta-\cos \theta \partial_{k_{\|}} \omega\right|$. Since the group velocity of the ion acoustic waves is small compared to $v_{\|} \cos \theta$, this will not affect the diffusion coefficient given in Eq. (18). The positions of the particles in the plane perpendicular to the magnetic field is not relevant in quasi-linear theory, and the parallel motion is properly described by our onedimensional model. Hence, as long as quasi-linear theory is valid, the heating rates we obtain are expected to apply also to a real, three-dimensional plasma.

Electron Landau damping is not included in the hybrid simulations presented here, which means that the growth rates are overestimated. The value found by Vaivads et al. (1995) was a factor of five lower, $\gamma \sim 6 \cdot 10^{-4} \Omega_{i}$, and this is probably more realistic. Still, if the parallel group velocity is estimated to $50 \mathrm{~km} / \mathrm{s}$, the wave energy density may grow by a factor of $10^{4}$ over $1000 \mathrm{~km}$ in altitude. These low frequency waves will then scatter the perpendicular velocities of the ions. When the wave amplitudes are about $6 \mathrm{mV} / \mathrm{m}$, the perpendicular temperature of the beam ions will increase by $30 \mathrm{eV} / \mathrm{s}$, or by $1 \mathrm{eV}$ in $23 \mathrm{~km}$ for a beam energy of $2.5 \mathrm{keV}$.

Our simulations indicate that the saturation of the fan instability mainly is caused by the increased perpendicular temperature of the beam ions. In the case we have studied, also the heating will essentially stop at a temperature of a few hundred $\mathrm{eV}$. The reason for this is that the ion gyro radius becomes comparable to the perpendicular wavelength. When the heating is due to resonance at the first harmonic of the ion gyrofrequency, the diffusion coefficient will vanish when the argument $k_{\perp} v_{\perp} / \Omega_{i}$ approaches the first zero of the Bessel function $J_{1}$ at about 3.8. Perpendicular temperatures of the order of $200 \mathrm{eV}$ are typical in observations of ion beams above the acceleration region, as would be expected from heating by waves with frequency below the ion gyrofrequency.

\section{Conclusions}

The growth rate of ion acoustic waves due to the ion fan instability and the rate of perpendicular heating caused by these waves as predicted by quasi-linear theory are confirmed quantitatively by our simulations. The fan instability will become important near the top of the auroral acceleration region, where the parallel electric field can no longer maintain a positive slope in the ion velocity distribution. Our results show that ion acoustic waves, even in the absence of a hump on the ion beam distribution, can grow to significant amplitudes within distances comparable to the acceleration region. These waves will efficiently heat the beam ions to perpendicular temperatures that are consistent with observations.

Acknowledgements. We would like to thank M. Yamauchi for the help in providing Viking data.

Topical Editor D. Alcaydé thanks A. W. Yau and Ph. Louarn for their help in evaluating this paper.

\section{References}

André, M., H. Koskinen, G. Gustafsson, and R. Lundin, Ion waves and upgoing ion beams observed by the Viking satellite, Geophys. Res. Lett., 14, 463-466, 1987.

Bahnsen, A., M. Jespersen, E. Ungstrup, R. Pottelette, M. Malingre, P. M. E. Décréau, M. Hamelin, H. de Feraudy, S. Perraut, and B. M. Pedersen, First Viking results: high frequency waves, Phys. Scr., 37, 469-474, 1988.

Bergmann, R., I. Roth, and M. K. Hudson, Linear stability of the $\mathrm{H}^{+}-\mathrm{O}^{+}$two-stream interaction in a magnetized plasma, $J$. Geophys. Res., 93, 4005-4020, 1988.

Birdsall C. K., and A. B. Langdon, Plasma physics via computer simulations, Adam Hilger, Bristol, 1991.

Cattell, C. A., F. S. Mozer, I. Roth, R. R. Anderson, R. C. Elphic, W. Lennartsson, and E. Ungstrup, ISEE 1 observations of electrostatic ion cyclotron waves in association with ion beams on auroral field lines from $\sim 2.5$ to $4.5 R_{E}, J$. Geophys. Res., 96, 11421-11439, 1991

Collin, H. L., W. K. Peterson, and E. G. Shelley, Solar cycle variation of some mass dependent characteristics of upflowing beams of terrestrial ions, J. Geophys. Res., 92, 4757-4762, 1987.

Fuchs, V., M. Shoucri, J. Teichmann, and A. Bers, Runaway electron distributions and their stability with respect to the anomalous Doppler resonance, Phys. Fluids, 31, 2221-2227, 1988.

Gorney, D. J., A. Clarke, D. Croley, J. Fennell, J. Luhmann, and P. Mizera, The distribution of ion beams and conics below 8000 km, J. Geophys. Res., 86, 83-89, 1981.

Gray, P. C., M. K. Hudson, R. Bergmann, and I. Roth, Simulation study of ion two-stream instability in the auroral acceleration region, Geophys. Res. Lett., 17, 1609-1612, 1990.

Gustafsson, G., M. André, L. Matson, and H. Koskinen, On waves below the local proton gyrofrequency in auroral acceleration regions, J. Geophys. Res., 95, 5889-5904, 1990.

Hultqvist, B., The swedish satellite project Viking, J. Geophys. Res., 95, 5749-5752, 1990.

Kaufmann, R. L., G. R. Ludlow, H. L. Collin, W. K. Peterson, and J. L. Burch, Interaction of upgoing auroral $\mathrm{H}^{+}$and $\mathrm{O}^{+}$beams, J. Geophys. Res., 91, 10080-10096, 1986.

Kondo, T., B. A. Whalen, A. W. Yau, and W. K. Peterson, Statistical analysis of upflowing ion beam and conic distributions at DE 1 altitudes, J. Geophys. Res., 95, 12091-12102, 1990.

Koskinen, H. E. J., R. Lundin, and B. Holback, On the plasma environment of solitary waves and weak double layers, $J$. Geophys. Res., 95, 5921-5929, 1990.

Miura, A., H. Okuda, and M. Ashour-Abdalla, Ion-beam-driven electrostatic ion cyclotron instabilities, Geophys. Res. Lett., 10, 353-356, 1983.

Okuda, H., J. M. Dawson, A. T. Lin, and C. C. Lin, Quasi-neutral particle simulation model with application to ion wave propagation, Phys. Fluids, 21, 476-482, 1978. 
Omelchenko, Yu. A., V. D. Shapiro, V. I. Shevchenko, M. AshourAbdalla, and D. Schriver, Modified lower hybrid fan instability excited by precipitating auroral electrons, J. Geophys. Res., 99, 5965-5975, 1994.

Reiff, P. H., H. L. Collin, J. D. Craven, J. L. Burch, J. D. Winningham, E. G. Shelley, L. A. Frank, and M. A. Friedman, Determination of auroral electrostatic potentials using highand low-altitude particle distributions, J. Geophys. Res., 93, 7441-7465, 1988.

Rönnmark, K., Computation of the dielectric tensor of a Maxwellian plasma, Plasma Phys., 25, 699-701, 1983.

Sandahl, I., R. Lundin, and L. Eliasson, The hot plasma spectrometers on Viking, KGI Preprint 077, Kiruna Geophysical Institute, 1985.

Schriver, D., M. Ashour-Abdalla, H. Collin, and N. Lallande, Ion beam heating in the auroral zone, J. Geophys. Res., 95, 10151028,1990 .
Shapiro, V. D., and V. I. Shevchenko, Astrophysical plasma turbulence. I, Sov. Sci. Rev. E. Astrophys Space Phys., 6, 425546, 1988

Vaivads, A., K. Rönnmark, and M. André, Generation of ion acoustic waves by fan instability, J. Geophys. Res., 100, 1943519440, 1995.

Wahlund, J.-E., P. Louarn, T. Chust, H. de Feraudy, A. Roux, B. Holback, P.-O. Dovner, and G. Holmgren, On ion acoustic turbulence and the nonlinear evolution of kinetic Alfvén waves in aurora, Geophys. Res. Lett., 21, 1831-1834, 1994.

Winglee, R. M., P. B. Dusenbery, H. L. Collin, C. S. Lin, and A. M. Persoon, Simulations and observations of heating of auroral ion beams, J. Geophys. Res., 94, 8943-8965, 1989.

Yau, A. W., B. A. Whalen, W. K. Peterson, and E. G. Shelley, Distribution of upflowing ionospheric ions in the high-altitude polar cap and auroral ionosphere, J. Geophys. Res., 89, 5507$5522,1984$. 